\title{
New trends in botulinum toxin use in dermatology
}

\author{
Stefania Guida ${ }^{1}$, Francesca Farnetani ${ }^{1}$, Steven P. Nisticò ${ }^{2}$, Caterina Giorgio Mariarosaria ${ }^{3}$, \\ Graziella Babino $^{3}$, Giovanni Pellacani ${ }^{1}$, Elisabetta Fulgione ${ }^{3}$
}

\begin{abstract}
1 Dermatology Unit, Department of Surgical, Medical, Dental and Morphological Sciences related to Transplant, Oncology and Regenerative Medicine, University of Modena and Reggio Emilia, Italy

2 Department of Health Sciences, University Magna Graecia, Catanzaro, Italy

3 Department of Dermatology, University of Campania Luigi Vanvitelli, Naples, Italy
\end{abstract}

Key words: botulinum toxin, BoNT-A, dermatology, hyperhidrosis, scars

Citation: Guida S, Farnetani F, Nisticò SP, Giorgio Mariarosaria C, Babino G, Pellacani G, Fulgione E. New trends in botulinum toxin use in dermatology. Dermatol Pract Concept. 2018;8(4):277-282. DOI: https://doi.org/10.5826/dpc.0804a05

Received: March 10, 2018; Accepted: May 28, 2018; Published: October 31, 2018

Copyright: $@ 2018$ Guida et al. This is an open-access article distributed under the terms of the Creative Commons Attribution License, which permits unrestricted use, distribution, and reproduction in any medium, provided the original author and source are credited.

Funding: None.

Competing interests: The authors have no conflicts of interest to disclose.

All authors have contributed significantly to this publication.

Corresponding author: Stefania Guida, MD, Department of Dermatology, University of Modena and Reggio Emilia, Via del Pozzo, 71, Modena, Italy. Email: drstefaniaguida@gmail.com

ABSTRACT Background: Recent studies have highlighted new botulinum neurotoxin (BoNT) applications in the field of dermatology.

Objective: To review current knowledge of BoNT use in dermatology.

Methods: The literature of the last 5 five years has been reviewed.

Results: We describe interesting protocols of BoNT treatment for hyperhidrosis (HH), hypertrophic scars and keloids, Raynaud phenomenon, facial flushing, oily skin, psoriasis, Hailey-Hailey disease, and cutaneous lesions like painful lesions and periorbital syringomas.

Conclusions: Several skin conditions eligible for BoNT treatment have been described. After the wide application for $\mathrm{HH}$ treatment, scars as well as vascular and inflammatory skin disorders, oily skin and cutaneous lesions represent fields of application of BoNT.

\section{Introduction}

The botulinum neurotoxin (BoNT) is produced by Clostridium botulinum bacteria. Seven toxin isoforms can be distinguished (BoNT A-G) [1,2], with BoNT-A and B being commercially available for clinical applications. Both BoNT$\mathrm{A}$ and BoNT-B are proteins composed of a heavy and a light chain. These chains are related to the mechanism of action of the toxin. Accordingly, the heavy chain binds the BoNT to the cholinergic nerve terminal while the light chain inhibits the release of acetylcholine from presynaptic vesicles $[3,4]$. The difference between BoNT-A and B is the type of protein the light chain cleaves and different procedures in which they are employed [5].

In 1989, BoNT, onabotulinumtoxinA, was initially approved for the treatment of strabismus and blepharospasm by the US Food and Drug Administration (FDA). Lately, aesthetic indications have been progressively intro- 
duced and the popularity of BoNT-A has increased $6956.6 \%$ from 1997 to 2016 [6].

The most recognized mechanism of action of BoNTs is the inhibition of neurotransmitters (acetylcholine, norepinephrine, substance P, calcitonin gene-related peptide [CGRP], and glutamate) release at the presynaptic neuromuscular junction. However, BoNTs can affect both sympathetic and parasympathetic functionality since acetylcholine is also a neurotransmitter of the autonomic nervous system [7]. Furthermore, recent evidence shows the effect of BoNTs on different human cell types, both neuronal and non-neuronal cells. These latter include epidermal keratinocytes, mesenchymal stem cells from subcutaneous adipose, neutrophils and macrophages, dermal fibroblasts, mast cells, sebocytes and vascular endothelial cells. Thus, several clinical applications are emerging in dermatology [2,8]. The aim of this article is to review new trends in BoNT treatment in dermatology.

\section{Methods}

Studies focusing on the use of BoNT in dermatology were retrieved from PubMed. We discovered 327 articles, from 2012 to December 2017, using the terms "botulinum toxin dermatology" and correlated $\mathrm{MeSH}$ terms ("botulinum toxins"[MeSH Terms] OR ("botulinum"[All Fields] AND "toxins"[All Fields]) OR "botulinum toxins"[All Fields] OR ("botulinum”[All Fields] AND "toxin”[All Fields]) OR "botulinum toxin"[All Fields]) AND ("dermatology”[MeSH Terms] OR “dermatology”[All Fields]). Of these articles, only 54 were included in this review. Exclusion criteria were: case reports, duplicated studies, papers focusing on other topics (ie, aesthetics, neurology), and articles written in languages other than English.

\section{Results}

\section{Hyperhidrosis}

Hyperhidrosis $(\mathrm{HH})$ is a skin condition characterized by excessive secretions of the eccrine glands located, above all, at the palms, soles and axillae, and it affects approximately $3 \%$ of the population. Thus treatment of hyperhidrosis with BoNT represents one of the most widespread applications of the BoNT. BoNT is injected intradermally in order to target sweat gland function through the inhibition of neurotransmission at nerve terminals reaching the glands [9-11].

Several issues regarding BoNT treatment have been addressed in the last few years. In detail, BoNT has been compared to other available treatment modalities. Treatment options should be carefully evaluated and selected for each patient $[7,12-16]$. However, BoNT has been proven to be effective in reducing the severity of $\mathrm{HH}$ and to improve quality of life (QoL) of $\mathrm{HH}$ patients. Accordingly, a profound QoL impairment is experienced by these patients, although no anxiety, depression or excessive alcohol consumption has been associated with the disease [17].

Both adults and children can benefit from BoNT-A and B treatment $[4,18,19]$.

Specifically, BoNT-B seems to be the treatment of choice in patients with multifocal $\mathrm{HH}$ since it can be used to treat multiple sites in the same session, thus improving QoL [4].

In pediatric patients, $\mathrm{HH}$ affects $1.6 \%$ of adolescents and $0.6 \%$ of prepubertal children [20], and it remains widely undertreated. However, BoNT has been demonstrated to be effective and safe [21]. A multicenter, nonrandomized, open-label study of onabotulinumtoxinA treatment (50 IU per axilla) of bilaterally primary axillary $\mathrm{HH}$ in adolescents from 12 to 17 years old showed an improvement of $75 \%$ in 79.4-93.2\% patients [22]. The efficacy of BoNT treatment has been proven for axillary $\mathrm{HH}$ by several randomized trials in the past. Therefore, BoNT for $\mathrm{HH}$ of the axillae is FDA-approved, but it has also been reported effective for the treatment of other sites. Palmar $\mathrm{HH}$, for instance, is usually treated with 100-200 IU. Pain can limit this treatment; thus several strategies have been evaluated to increase the compliance. Among these, ice with pressure, ice $<20$ or $>20$ seconds associated or not with topical anesthesia [23,24], general anesthesia, nerve blocks, vibration, pressure [21], and needle-free injections (Med-Jet MBX, Medical International Technologies, Montreal, QC, Canada) [25]. However, an open-label prospective study involving 20 patients with palmar $\mathrm{HH}$, treated with needle injection on the right hand and with a needle-free device, has proven the efficacy of the device to be lower as compared to the classic needle injection [25].

Repeated injection protocols have been proposed for both axillary and palmar treatment, in 3 different retrospective studies [26-28]. Repeated injections have been demonstrated to increase the duration of the effect from 5.5 to 8.5 months with 125 IU of abobotulinumtoxinA per underarm while it passed from 4 to 5 months when $50 \mathrm{U}$ of onabotulinumtoxinA per axilla were employed [26, 27]. The median duration of efficacy for palmar repetitive treatment was 7 months for the first injection and 9.5 months for the last with $250 \mathrm{IU}$ of abobotulinumtoxinA per palm [28]. Palmar treatment should be performed carefully on the thenar eminence, as there is a risk of inducing weakness of the hand. On the other hand, repeated injections can be related to the development of neutralizing antibodies. Nevertheless, a 3-month interval between each injection has not been related to the presence of these antibodies [26,28].

Data concerning plantar treatment with BoNT are scarce [29]. Doses employed vary from 50 to 250 IU of onabotulinumtoxinA per plantar area with effects lasting 3-6 months [30]. 
Indications for $\mathrm{HH}$ involving other cutaneous sites and osmidrosis treatment have also been described [31-36].

\section{Hypertrophic Scars and Keloids}

BoNT treatment has been proposed for the treatment of hypertrophic scars and keloids [37,38]. Tension due to motion is a well-known factor in determining scar tissue hypertrophy [39]. Thus, the ability of BoNT to reduce muscle contraction in the scar area can result in a decrease of skin tension, microtrauma, and subsequent inflammation [39]. Another underlying biological mechanism that has been hypothesized is the contribution in reducing the expression of transforming growth factor beta, the main regulator of hypertrophic scar formation [40]. Injection of BoNT may vary from 17.540 IU of onabotulinumtoxinA. BoNT can be employed either at the site of scar formation or suture removal [41].

A split-scar, double-blind randomized controlled trial involving 15 patients with early post-thyroidectomy scars revealed a significant improvement in one half of the scar, corresponding to the BoNT-treated area, as compared to the other part injected with $0.9 \%$ saline solution. Treatments were performed within 10 days after the surgery and evaluations were performed after 6 months [42].

Keloids are commonly managed with intralesional corticosteroids. A recent randomized controlled trial enrolled 24 patients with keloids randomly treated with intralesional steroids and BoNT-A. Both treatments were effective in reducing the volume and height of lesions and increasing their softening. Nevertheless, BoNT was more effective in reducing itching and pain related to keloids [43]. However, 3D profilometry used to objectively evaluate BoNT-A-treated keloids revealed the absence of results in some cases. This clinical observation has been supported by studies showing no TGF beta or fibroblast variations after BoNT treatment of scars. Consequently, contradictory results from a clinical and biological point of view are available at the moment, and further well-designed studies are necessary to evaluate the real role of BoNT in the scarring process [5].

\section{Raynaud Phenomenon}

Raynaud phenomenon (RP) consists of severe vasospasms leading to pain and digital ulceration, thus impairing daily activities [44]. Behavioral lifestyle variations and medical treatment enable heterogeneous results. The role of BoNT treatment has been emerging in the last decades. Recently, BoNT-B has been used to treat Raynaud's phenomenon and digital ulcers in 45 patients with systemic sclerosis. Patients were randomly divided into 4 groups: a control group receiving no treatment, and 3 treatment groups using increasing units of BoNT-B (250, 1,000 or 2,000 IU). The hand with more severe symptoms was treated. Results of this study highlight the improvement in terms of both Raynaud's score (for 16 weeks after treatment) and the number of digital ulcers in patients treated with 1,000 and 2,000 IU of BoNT$\mathrm{B}$, as compared to the other 2 groups [45]. Furthermore, BoNT-A and B treatments have been proven to improve pulp temperature in patients with Raynaud, with or without systemic sclerosis $[44,45]$.

\section{Facial Flushing}

Facial flushing consists of an episode of redness associated with a burning sensation. It can be primary or idiopathic and secondary to rosacea or hormonal stimuli like menopause. BoNT has been proven to provide symptomatic relief in patients with facial flushing through the inhibition of acetylcholine signaling pathway [46]. An open-label randomized controlled trial involving 24 patients with facial flushing showed an improvement within 2 to 3 weeks after the treatment. BoNT-A was injected, $1 \mathrm{IU}$ per $\mathrm{cm}^{2}$, for a total amount of $30 \mathrm{IU}$ in one session [47]. However, other protocols have also been described. The injection of 100 IU of onabotulinumtoxinA diluted in $7 \mathrm{~mL}$ of saline solution, distributed in microdroplets $(0.05 \mathrm{~mL})$, has been reported to be effective [48].

A clinical trial involving 25 patients with facial erythema of erythematotelangiectatic rosacea was performed. The nasal tip, nasal bridge, and nasal ala of 15 patients completing the study were treated with abobotulinumtoxinA 15-45 IU. The assessment of clinical response followed a standardized grading system $(0=$ absent, $1=$ mild erythema, $2=$ moderate erythema, and 3 = severe erythema), revealing a significant improvement in erythema grade, as compared to baseline, at 1,2 , and 3 months after treatment $(\mathrm{P}<.05, \mathrm{P}<.001$, and $\mathrm{P}<.05$, respectively) [49]. Although BoNT may cause headache, albeit rarely, it can be considered an option for reducing the severity of flushing $[46,50]$. However, studies with longterm follow-up are lacking [5].

\section{Oily Skin}

Oily skin is a common disorder, and treatment options often provide unsatisfactory results. The basis for BoNT-A treatment of oily skin, providing a reduction in sebum production, seems to lie in the expression of muscarinic acetylcholine receptors in sebaceous glands. These receptors control sebocyte differentiation and sebum production [3].

After preliminary data obtained on sebum reduction in a retrospective study, a prospective study involving 25 patients showing oily skin on the forehead was performed. Patients were treated with 10 intradermal injections of a total amount of 30-45 IU of abobotulinumtoxinA has been performed. A Sebumeter (Courage + Khazaka Electronic GmbH, Cologne, Germany) was used to assess baseline and post-treatment 
sebum production. All 23 patients completing the study were satisfied after the treatment, with variable degrees of satisfaction (21 were satisfied, 1 was very satisfied, and 1 somewhat satisfied). However, Sebumeter readings revealed a significant sebum reduction in all patients at 1 week and 1 , 2 , and 3 months $(\mathrm{P}<0.001)$ [51]. Further studies are needed.

\section{Psoriasis}

The basis for BoNT-A use in psoriasis can be related to the inhibited release of substance P and CGRP. In particular, inverse psoriasis has been proven to benefit from BoNT, reducing both itch and pain. Intralesional BoNT-A has been employed for intertriginous psoriasis with the aim of reducing local sweating, skin maceration, and consequent infection. Subjective improvement of skin lesions was found in $87 \%$ of 15 patients injected with a total of 50100 IU of onabotulinumtoxinA per patient, depending on the extent and severity of lesions, with $2.4 \mathrm{IU}$ per point and $2.8 \mathrm{~cm}$ apart [52].

No placebo-controlled studies are available at the moment, thus impairing a clear evaluation of the efficacy of BoNT in this disease [5].

\section{Hailey-Hailey Disease}

Similarly to what has been previously described for psoriasis, acanthosis and symptomatic relief seem to be the main effects of BoNT treatment in Hailey-Hailey disease. However, associated costs for achieving only temporary relief are a major issue [53]

\section{Cutaneous Lesions}

Pain relief has been evaluated in 18 patients with painful cutaneous leiomyomas who were randomized to obtain either onabotulinumtoxinA treatment versus placebo. BoNT-A was proven to be effective in pain improvement [54].

Periorbital syringomas are commonly treated with CO2 laser. However, the combined approach with onabotulinumtoxinA has been found to give better results in 48 patients, as compared to 44 subjects treated with CO2 only [55].

\section{Conclusions}

In conclusion, we describe innovative protocols and applications for BoNT use in dermatology, reviewing the literature of the last 5 years. Although the action of this drug has a complex mechanism, the effect of BoNT on many cellular types has been highlighted. Based on these data, several applications of BoNT have emerged. After the wide application for $\mathrm{HH}$ treatment, scars, as well as vascular and inflammatory skin disorders, oily skin and cutaneous lesions seem to be interesting fields of application of BoNT. On the other hand, there are some factors limiting BoNT use, such as the high cost of the well-tolerated BoNT-A for chronic skin conditions.

Further studies are needed to improve the knowledge of the connection between BoNT and the cutaneous neuroimmune system and to better define standard protocols of treatment.

\section{References}

1. Motegi SI, Sekiguchi A, Saito S, et al. Successful treatment of Raynaud's phenomenon and digital ulcers in systemic sclerosis patients with botulinum toxin B injection: assessment of peripheral vascular disorder by angiography and dermoscopic image of nail fold capillary. J Dermatol. 2017. [Epub ahead of print]. doi: 10.1111/1346-8138.14140

2. Grando SA, Zachary CB. The non-neuronal and non-muscular effects of botulinum toxin: a graceful opportunity for a deadly molecule to treat a human disease in the skin and beyond. $\mathrm{Br} \mathrm{J}$ Dermatol. 2017. [Epub ahead of print]. doi: 10.1111/bjd.16080

3. Endly DC, Miller RA. Oily Skin: a review of treatment options. $J$ Clin Aesthet Dermatol. 2017;10(8):49-55.

4. Eriksson Mirkovic S, Rystedt A, Balling M, Swartling C. Hyperhidrosis substantially reduces quality of life in children: a retrospective study describing symptoms, consequences and treatment with botulinum toxin. Acta Derm Venereol. 2017. [Epub ahead of print]. doi: 10.2340/00015555-2755

5. Campanati A, Martina E, Giuliodori K, Consales V, Bobyr I, Offidani A. Botulinum toxin off-label use in dermatology: a review. Skin Appendage Disord. 2017;3(1):39-56.

6. The American Society for Aesthetic Plastic Surgery https://www. surgery.org/sites/default/files/ASAPS-Stats2016.pdf Accessed 31 Dec 2017

7. Budamakuntla L, Loganathan E, George A, Revanth BN, Sankeerth V, Sarvjnamurthy SA. Comparative study of efficacy and safety of botulinum toxin A injections and subcutaneous curettage in the treatment of axillary hyperhidrosis. J Cutan Aesthet Surg. 2017;10(1):33-39.

8. Kim YS, Hong ES, Kim HS. Botulinum toxin in the field of dermatology: novel indications. Toxins (Basel). 2017;9(12).

9. D'Epiro S, Macaluso L, Salvi M, et al. Safety and prolonged efficacy of botulin toxin A in primary hyperhidrosis. Clin Ter. 2014;165(6):e395-400.

10. de Almeida AR, Montagner S. Botulinum toxin for axillary hyperhidrosis. Dermatol Clin. 2014;32(4):495-504.

11. Brown AL, Gordon J, Hill S. Hyperhidrosis: review of recent advances and new therapeutic options for primary hyperhidrosis. Curr Opin Pediatr. 2014;26(4):460-465.

12. Nasr MW, Jabbour SF, Haber RN, Kechichian EG, El Hachem L. Comparison of microwave ablation, botulinum toxin injection, and liposuction-curettage in the treatment of axillary hyperhidrosis: a systematic review. J Cosmet Laser Ther. 2017;19(1):36-42.

13. An JS, Hyun Won C, Si Han J, Park HS, Seo KK. Comparison of onabotulinumtoxinA and rimabotulinumtoxinB for the treatment of axillary hyperhidrosis. Dermatol Surg. 2015;41(8):960-967.

14. Rajagopal R, Mallya NB. Comparative evaluation of botulinum toxin versus iontophoresis with topical aluminum chloride hexahydrate in treatment of palmar hyperhidrosis. Med J Armed Forces India. 2014;70(3):247-252. 
15. Alvarez MA, Ruano J, Gómez FJ, et al. Differences between objective efficacy and perceived efficacy in patients with palmar hyperhidrosis treated with either botulinum toxin or endoscopic thoracic sympathectomy. J Eur Acad Dermatol Venereol. 2013;27(3):e282-288.

16. Ibrahim O, Kakar R, Bolotin D, et al. The comparative effectiveness of suction-curettage and onabotulinumtoxin-A injections for the treatment of primary focal axillary hyperhidrosis: a randomized control trial. J Am Acad Dermatol. 2013;69(1):88-95.

17. Shayesteh A, Boman J, Janlert U, Brulin C, Nylander E. Primary hyperhidrosis: implications on symptoms, daily life, health and alcohol consumption when treated with botulinum toxin. J Dermatol. 2016;43(8):928-933.

18. Kouris A, Armyra K, Christodoulou C, Karimali P, Karypidis D, Kontochristopoulos G. Quality of life in patients with focal hyperhidrosis before and after treatment with botulinum toxin A. ISRN Dermatol. 2014;2014:308650.

19. Rosell K, Hymnelius K, Swartling C. Botulinum toxin type A and $\mathrm{B}$ improve quality of life in patients with axillary and palmar hyperhidrosis. Acta Derm Venereol. 2013;93(3):335-339.

20. Bohaty BR, Hebert AA. Special considerations for children with hyperhidrosis. Dermatol Clin. 2014;32(4):477-484.

21. Gordon JR, Hill SE. Update on pediatric hyperhidrosis. Dermatol Ther. 2013;26(6):452-461.

22. Glaser DA, Pariser DM, Hebert AA, et al. A Prospective, nonrandomized, open-label study of the efficacy and safety of onabotulinumtoxinA in adolescents with primary axillary hyperhidrosis. Pediatr Dermatol. 2015;32(5):609-17.

23. Dixit $S$, Lowe P, Fischer G, Lim A. Ice anaesthesia in procedural dermatology. Australas J Dermatol. 2013;54(4):273-276.

24. Kang A, Burns E, Glaser DA. Botulinum toxin A for palmar hyperhidrosis: associated pain, duration, and reasons for discontinuation of therapy. Dermatol Surg. 2015;41(2):297-298.

25. Vadeboncoeur S, Richer V, Nantel-Battista M, Benohanian A. Treatment of palmar hyperhidrosis with needle injection versus low-pressure needle-free jet injection of onabotulinumtoxina: an open-label prospective study. Dermatol Surg. 2017;43(2):264269.

26. Lecouflet M, Leux C, Fenot M, Célerier P, Maillard H. Duration of efficacy increases with the repetition of botulinum toxin A injections in primary axillary hyperhidrosis: a study in 83 patients. J Am Acad Dermatol. 2013;69(6):960-964.

27. Brehmer F, Lockmann A, Grönemeyer LL, Kretschmer L, Schön MP, Thoms KM. Repetitive injections of botulinum toxin A continuously increase the duration of efficacy in primary axillary hyperhidrosis: a retrospective analysis in 101 patients. J Dtsch Dermatol Ges. 2015;13(8):799-805.

28. Lecouflet M, Leux C, Fenot M, Célerier P, Maillard H. Duration of efficacy increases with the repetition of botulinum toxin $\mathrm{A}$ injections in primary palmar hyperhidrosis: a study of 28 patients. J Am Acad Dermatol. 2014;70(6):1083-1087.

29. Weinberg T, Solish N, Murray C. Botulinum neurotoxin treatment of palmar and plantar hyperhidrosis. Dermatol Clin. 2014;32(4):505-515.

30. Hosp C, Naumann MK, Hamm H. Botulinum toxin treatment of autonomic disorders: focal hyperhidrosis and sialorrhea. Semin Neurol. 2016;36(1):20-28.

31. Karlsson-Groth A, Rystedt A, Swartling C. Treatment of compensatory hyperhidrosis after sympathectomy with botulinum and anticholinergics. Clin Auton Res. 2015;25(3):161-167.
32. Lezanski-Gujda A, Bingham JL, Logemann NF. Botulinum toxin: An effective treatment for prosthesis-related hyperhidrosis in patients with traumatic amputations. Indian Dermatol Online J. 2015;6(1):1-3

33. Hexsel D, Soirefmann M, Porto MD, Schilling-Souza J, Siega C. Fields of anhidrotic effects of onabotulinumtoxinA in patients with compensatory hyperhidrosis. Dermatol Surg. 2015;41 Suppl 1:S93-100.

34. Glaser DA, Galperin TA. Botulinum toxin for hyperhidrosis of areas other than the axillae and palms/soles. Dermatol Clin. 2014;32(4):517-525.

35. Xie A, Nie L, Tan Q. Local injection of botulinum toxin A: an alternative therapy for axillary osmidrosis. J Dermatol. 2014 Feb;41(2):153-156.

36. Karlquist M, Rosell K, Rystedt A, Hymnelius K, Swartling C. Botulinum toxin B in the treatment of craniofacial hyperhidrosis. J Eur Acad Dermatol Venereol. 2014;28(10):1313-1317.

37. Heppt MV, Breuninger H, Reinholz M, Feller-Heppt G, Ruzicka T, Gauglitz GG. Current strategies in the treatment of scars and keloids. Facial Plast Surg. 2015;31(4):386-95.

38. Arno AI, Gauglitz GG, Barret JP, Jeschke MG. Up-to-date approach to manage keloids and hypertrophic scars: a useful guide. Burns. 2014;40(7):1255-1266.

39. Gauglitz GG. Management of keloids and hypertrophic scars: current and emerging options. Clin Cosmet Investig Dermatol. 2013;6:103-114.

40. Elhefnawy AM. Assessment of intralesional injection of botulinum toxin type A injection for hypertrophic scars. Indian J Dermatol Venereol Leprol. 2016;82(3):279-83.

41. Forbat E, Ali FR, Al-Niaimi F. Non-cosmetic dermatological uses of botulinum neurotoxin. J Eur Acad Dermatol Venereol. 2016;30(12):2023-2029.

42. Kim YS, Lee HJ, Cho SH, Lee JD, Kim HS. Early postoperative treatment of thyroidectomy scars using botulinum toxin: a splitscar, double-blind randomized controlled trial. Wound Repair Regen. 2014;22(5):605-612.

43. Shaarawy E, Hegazy RA, Abdel Hay RM. Intralesional botulinum toxin type A equally effective and better tolerated than intralesional steroid in the treatment of keloids: a randomized controlled trial. J Cosmet Dermatol. 2015;14(2):161-166.

44. Jenkins SN, Neyman KM, Veledar E, Chen SC. A pilot study evaluating the efficacy of botulinum toxin A in the treatment of Raynaud phenomenon. J Am Acad Dermatol. 2013;69(5):834835.

45. Motegi SI, Uehara A, Yamada K, et al. Efficacy of botulinum toxin $\mathrm{B}$ injection for Raynaud's phenomenon and digital ulcers in patients with systemic sclerosis. Acta Derm Venereol. 2017;97(7):843-850.

46. Abokwidir M, Feldman SR. Rosacea management. Skin Appendage Disord. 2016;2(1-2):26-34.

47. Eshghi G, Khezrian L, Alirezaei P. Botulinum toxin A in treatment of facial flushing. Acta Med Iran. 2016;54(7):454-457.

48. Weinkle AP, Doktor V, Emer J. Update on the management of rosacea. Clin Cosmet Investig Dermatol. 2015;8:159-177.

49. Bloom BS, Payongayong L, Mourin A, Goldberg DJ. Impact of intradermal abobotulinumtoxinA on facial erythema of rosacea. Dermatol Surg. 2015;41 Suppl 1:S9-16.

50. Weinkle AP, Doktor V, Emer J. Update on the management of rosacea. Plast Surg Nurs. 2015;35(4):184-202. 
51. Rose AE, Goldberg DJ. Safety and efficacy of intradermal injection of botulinum toxin for the treatment of oily skin. Dermatol Surg. 2013;39(3 Pt 1):443-448.

52. Wang TS, Tsai TF. Intralesional therapy for psoriasis. J Dermatol Treat. 2013;24(5):340-247.

53. Farahnik B, Blattner CM, Mortazie MB, Perry BM, Lear W, Elston DM. Interventional treatments for Hailey-Hailey disease. J Am Acad Dermatol. 2017;76(3):551-558.e3.
54. Naik HB, Steinberg SM, Middelton LA, et al. Efficacy of intralesional botulinum toxin A for treatment of painful cutaneous leiomyomas: a randomized clinical trial. JAMA Dermatol. 2015;151(10):1096-102.

55. Seo HM, Choi JY, Min J, Kim WS. Carbon dioxide laser combined with botulinum toxin A for patients with periorbital syringomas. J Cosmet Laser Ther. 2016;18(3):149-153. 